\title{
Finite element analysis of mini-plate stabilization of human mandible angle fracture - a comparative study
}

\author{
PIOTR WĄDOŁOWSKI*1 ${ }^{1}$, GRZEGORZ KRZESIŃSKI ${ }^{1}$, PIOTR GUTOWSKI ${ }^{2}$ \\ ${ }^{1}$ Warsaw University of Technology, Faculty of Power and Aeronautical Engineering, \\ Division of Strength of Materials and Structures, Warsaw, Poland. \\ ${ }^{2}$ Medical University of Warsaw, Warsaw, Poland.
}

\begin{abstract}
Purpose: The purpose of this study was to analyze three patterns of mandible angle fracture treatment by means of the finite element analysis. Methods: Investigation has been based on the mandible geometry reconstructed with use of hospitalized patient CT data. The KLS Martin mini-plates with corresponding screws were used to establish proper fracture stabilization. Models were run assuming isotropic and elasto-plastic material properties of connecting devices and cortical bone. The main masticatory muscles and artificial temporomandibular joint have been incorporated to assure mandible physiological movement. The gage loading has been applied in three different locations to cover wider range of possible mastication loading cases during daily routine. A different contact conditions have been applied to the fracture plane to simulate both load bearing and sharing behaviors. Prepared FEM models reflect the most frequently used surgery's approaches to mandible angle fracture treatment. A specific nomenclature has been introduced to describe particular model. The tension plate, with one connecting mini-plate, two-point fixation and combined fixation, both using two mini-plates respectively. Results: Performed analysis allowed for a detailed estimation of the mini-plate connection response under the applied gauge loading. The equivalent stress within the mini-plates and surrounding cortical bone have been compared between all models. Regarding the fracture plane, the contact status and pressure have been considered. Conclusions: The combined fixation model, acting as a biplanar fastener system, presents the highest flexibility and connection efficiency.
\end{abstract}

Key words: finite element analysis, maxillofacial surgery, mandibular angle fracture, mini-plate osteosynthesis, contact mechanics

\section{Introduction}

Fractures of mandible bone are among the most frequent facial injuries [6], [18] [24]. Following the literature information, the etiology depends on several factors such as patient ancestry, sex, age or lifestyle [18]. Common causes of mandibular fractures are road traffic accidents, assaults, falls, sport and work-related injuries [6]. The mandible bone is more frequently fractured comparing to the other facial bones due to its prominence within the facial skeleton. Mandible, the strongest facial bone, has fairly complex architec- ture, reminding archery bow, where the central part is stiffer compared to the ends. Rotation around the transverse axis during the masticatory process is supported by the temporomandibular joint. Mandible fractures could be divided into particular groups depending on fracture location. Fractures mainly occur at the condylar neck, the mandible angle and the symphysis [18]. Mandible fractures could be treated using open or closed reduction, depending on fracture type and location [9]. The aim of the treatment is to provide required stability of fracture region during the treatment period with a minimum impact on the surrounding bone and soft tissues. A mini-plate osteo-

\footnotetext{
* Corresponding author: Piotr Wądołowski, Faculty of Power and Aeronautical Engineering, Warsaw University of Technology, ul. Nowowiejska 24, 00-665, Warsaw, Poland. E-mail: pwadolowski@meil.pw.edu.pl

Received: April 7th, 2020

Accepted for publication: June 16th, 2020
} 
synthesis technique is a modern type of bone internal fixation using metal plates connected to the fractured bone by a set of screws, widely used to mandible trauma treatment. Compared to the previously used intermaxillary fixation, such as transosseous wiring, the mini-plate connection provides adequate stability, a shorter period of hospitalization and is more convenient for daily operation.

The finite element analysis that has been widely used in engineering mechanics enables the medic to investigate different problems of both dental and maxillofacial surgery including highly detailed analysis of miniplate behavior using various types of materials, geometry of the devices, loads and boundary conditions [5], [15], [22]. Flexibility of FE models makes it possible to study different configurations including miniplate position and geometry. Finding the optimized mechanical stabilization of the bone fracture is important from the medical point of view because of the strong correlation between the osteosynthesis technique and stress distribution around the region of fracture, and, finally, the treatment results. Available literature describes a number of different approaches to numerical analysis of mandible fracture treatment. This phenomenon was used to analyze by the FEM method at the beginning of the present century [10]. Mandible geometries were mainly obtained from a 3D scans [24] or manual measurements of dry bone [10]. The newest approach is to generate the detailed bone geometry from the Computed Tomography (CT) scans [14], [19], [22]. A high-quality geometry is easier to operate and better reflects the physiological shape. Furthermore, the CT helps to precisely split the mandible into the cortical and cancellous bones based on the Hounsfield's scale [11]. Material models used to conduct mechanical analysis assume mainly the simplified isotropic homogenous properties of both the cortical and the cancellous bones [22]. That assumption may result from the lack of detailed material data or just from the FEM model purposes. In many cases, the entire model or connection type behavior is more important from the analysis standpoint rather than the exact stress or strain value. Mechanical properties of mandible bone vary through the population, so it is not possible to cover all existing material scenarios, but for the very detailed analysis it is mandatory to employ the orthotropy material models. Works of Schwartz-Dabney and Dechow [23] enable the researchers to apply the orthotropic material properties for particular mandible parts, but not many works have taken it into consideration [11].

The purpose of this study was to analyze the impact of mini-plate fixation type, fracture plane contact conditions and loading location, based on the stress distribution within the mini-plate and the surrounding cortical bone. Achieved results can provide the biomechanical guidelines for the mini-plate applications and allow to decrease the amount of postoperative complications.

\section{Materials and methods}

\subsection{Mandible geometry model preparation}

The investigated mandible bone geometry was obtained from the patient hospitalized in the Department of Cranio-Maxillo-Facial Surgery, Medical University of Warsaw. The fracture occurred in the mandible angle region on the left side with a significant bone translation and was a result of interpersonal violence (Fig. 1A). The mandible bone was scanned by computed tomography before the treatment in the
A)

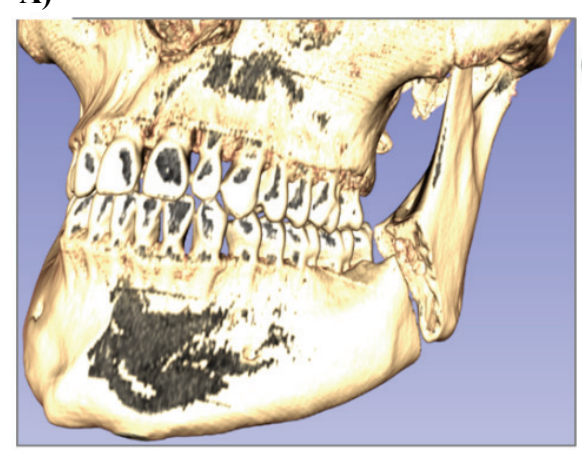

B)

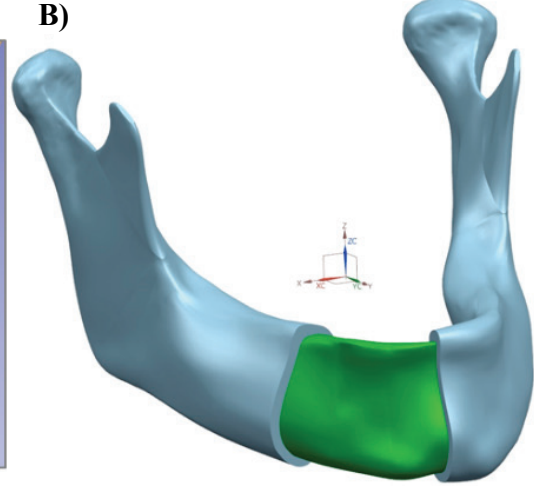

C)

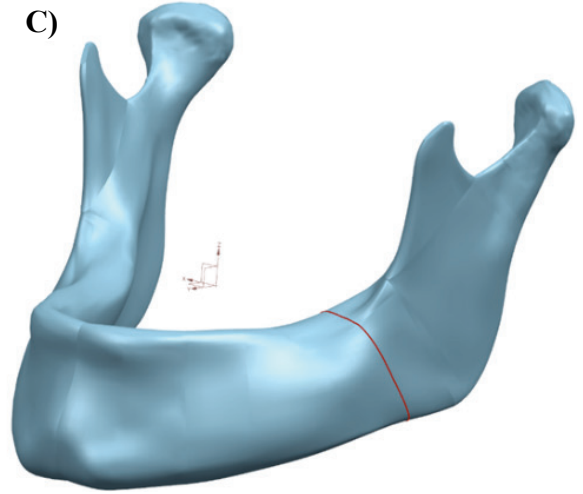

Fig. 1. (A) CT scan of hospitalized patient before the treatment,

(B) Positioned mandible solid model consisting of cortical (grey) and cancellous (green) layers,

(C) Fracture line location (red line) 
axial direction at $0.8 \mathrm{~mm}$ intervals. The DICOM data from the conducted scans was imported by the 3D Slicer ${ }^{\circledR}$ free software to separate the considered bone from soft tissues, cartilages and adjacent bones. Selected data was transformed to the stereolithographic (STL) format, which allowed for further modification. Created model was positioned in the Cartesian coordinate system along the main axis for easy handling during the next steps.

Due to the poor CT data quality, the external surfaces were cleaned from the geometrical artefacts, small holes and spikes by using the healing tool of Siemens NX software, where imported geometry has been cut by a set of planes parallel to the frontal plane to create the intersection curve model.

Work on these curves allows to recreate both, cortical and cancellous, bone layers with appropriate thickness throughout the model (Fig. 1B). The teeth geometry including the enamel, dentine and periodontal ligament was neglected due to the negligible impact on the stress distribution of the mandible fracture [21], [22]. The fracture was simulated by the cut plane on the left side of the mandible angle, covering the fracture location of the hospitalized patient (Fig. 1C). This paper considers three connection patterns. First of them (Fig. 2A), assumes one mini-plate at the middle region of distal mandible surface. The second one (Fig. 2B), contains two mini-plates located at the top and bottom region of outer mandible surface. Mini-plates are nearly parallel to each other. The third solution (Fig. 2C), consists of two mini-plates, where the lower overlap the previous lower plate location and the top plate is located at the oblique line. All the connecting plates were applied perpendicularly and symmetrically to the fracture line. These three approaches have been selected in accordance with the maxillofacial surgeon's experience as the most applicable cases during the mandible fracture treatment. All presented models were prepared to the testing of mechanical strength and stress validation within the connecting miniplates.

\subsection{Mini-plate and connecting screws geometry models}

The 3D miniplate geometry was based on the commercial KLS MARTIN System Mini 2.0, a standard, non-compression titanium mini-plate without the locking system that has been widely used in the medical practice. The mini-plate has a thickness of $1 \mathrm{~mm}$ and comprises of four screw application holes. The cooperating screws, CentreDrive System Mini 2.0, commonly used to locking mentioned mini-plates were selected for the created model.

During the CAD model preparation, screws have been simplified to neglect small features, like a wrench socket and a top chamfer which have not visibly impacted the executed analysis. The thread was simplified to cylindrical surface assumed to be surrounded by the cortical and cancellous bones. It was shown [8] that there is a negligible difference between threaded and simplified screws (cylindrical surface instead of helical structure) regarding the stress distribution within the surrounding bone in a macro scale.

\subsection{Material properties}

Following the KLS Martin material specification, an isotropic pure commercial titanium CP-Ti - UNS R50700 was assigned to the mini-plate involved in this report, while the screws were made from a titanium alloy Ti-6Al-4V Extra Low Interstitials. To cover the elastic-plastic behavior, for both materials, the experimental stress-strain curves were used. According to the [4], CP-Ti grade 4 - UNS R50700 has yielded tensile strength at the level of $480 \mathrm{MPa}$. The [7] provides the value of yield tensile strength for Ti-6Al-4V ELI, UNS R56401 is at the level of $880 \mathrm{MPa}$. Isotropic and homogenous properties were assumed for both cortical and cancellous bones. The Young's modulus, the Poisson's ratio and experimental stress-
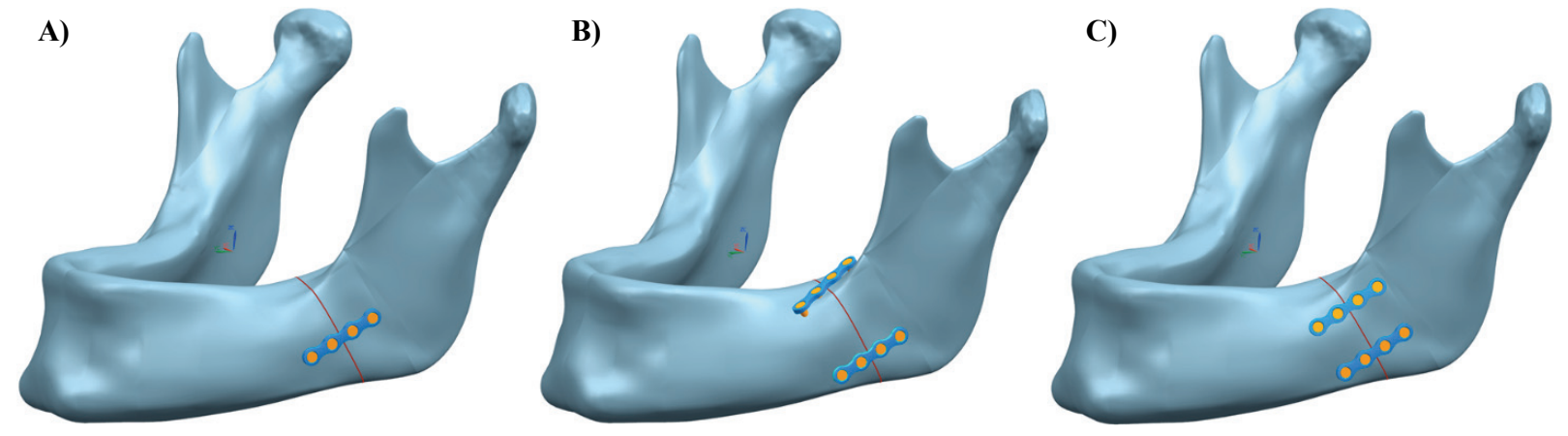

Fig. 2. Investigated connecting approaches 
Table 1. Material properties

\begin{tabular}{|l|c|c|c|c|}
\hline \multicolumn{1}{|c|}{ Component } & $\begin{array}{c}\text { Young's modulus (E) } \\
{[\mathrm{GPa}]}\end{array}$ & $\begin{array}{c}\text { Poisson's ratio }(v) \\
{[-]}\end{array}$ & $\begin{array}{c}\text { Yield strength } \\
{[\mathrm{MPa}]}\end{array}$ & $\begin{array}{c}\text { Ultimate tensile strength } \\
{[\mathrm{MPa}]}\end{array}$ \\
\hline Miniplate (CP-Ti) & 185 & 0.37 & 480 & 691 \\
\hline $\begin{array}{l}\text { Screws } \\
\text { (Ti-6Al-4V ELI) }\end{array}$ & 113.8 & 0.342 & 880 & 1011 \\
\hline Bone (cortical) & 13.7 & 0.3 & 108 & 130 \\
\hline Bone (cancellous) & 1.6 & 0.3 & - & - \\
\hline Muscles & 0.1 & 0.45 & - & - \\
\hline Artificial TMJ & 0.1 & 0.3 & - & - \\
\hline
\end{tabular}

train curve were obtained from the literature [20], [23]. The use of isotropic material properties is a strong simplification for the stiffness-variable mandible bone, but for the connection type or initial preload back-toback comparison purposes, hereby described, it seems to be correct assumption. Similar deltas are anticipated for the mandible's orthotropic material due to its linear behavior. A mastication muscle system can be modeled as active or passive structures. The active system, mainly considered in the literature, consists of a set of force vectors with appropriate magnitude and direction oriented towards the direction of particular muscle action [11]. The passive system, undertaken in this work, acts as an elastic shell surfaces mimicking muscles. This approach requires information about the cross-section area, permissible transferred masticatory loading and the acting length of particular muscle. It assumes that the force within the muscle is proportional to its physiological stiffness. Based on the maximum tension and cross-section area provided by [13], [17], the average Young's modulus of $0.1 \mathrm{GPa}$ and Poisson ratio of 0.45 have been assigned to all muscles. The material for artificial TMJ was selected using the sensitive study to stabilize the system, assure free rotation of mandible about the traverse axis and to avoid stress concentration at the mandible neck [13]. All of the material constants are included in the Table 1.

\subsection{FEM model}

The assembly of the treated mandible, muscles and connecting devices were imported into the ANSYS software version 17.1 to generate the discrete FEM models. Three types of finite elements, SOLID 186, SOLID 187 and SHELL 181 were used. Both, the SOLID186 and SOLID187, are higher order 3D solid elements that exhibit quadratic displacement behavior [1]. Based on the engineering experience, the structural grid consists of hexahedral elements, which provides more accurate results, compared to free meshes composed of tetrahedral elements. Thus, Solid186 was used to generation structural meshes of mini-plates, screws and bone regions, where the high stress gradient is expected. Solid187 is well-suited to modeling irregular meshes and was used to create the mandible cortical and cancellous bones and the TMJ mimic block. It was used as variable tetrahedral element size within both, the cortical and the cancellous bones, depending on bone thickness and locations of possible high stress gradient. The SHELL181 elements were used to model structural grid of the muscle system. The shell layer thickness was set to match the average cross-section area for a particular muscle.
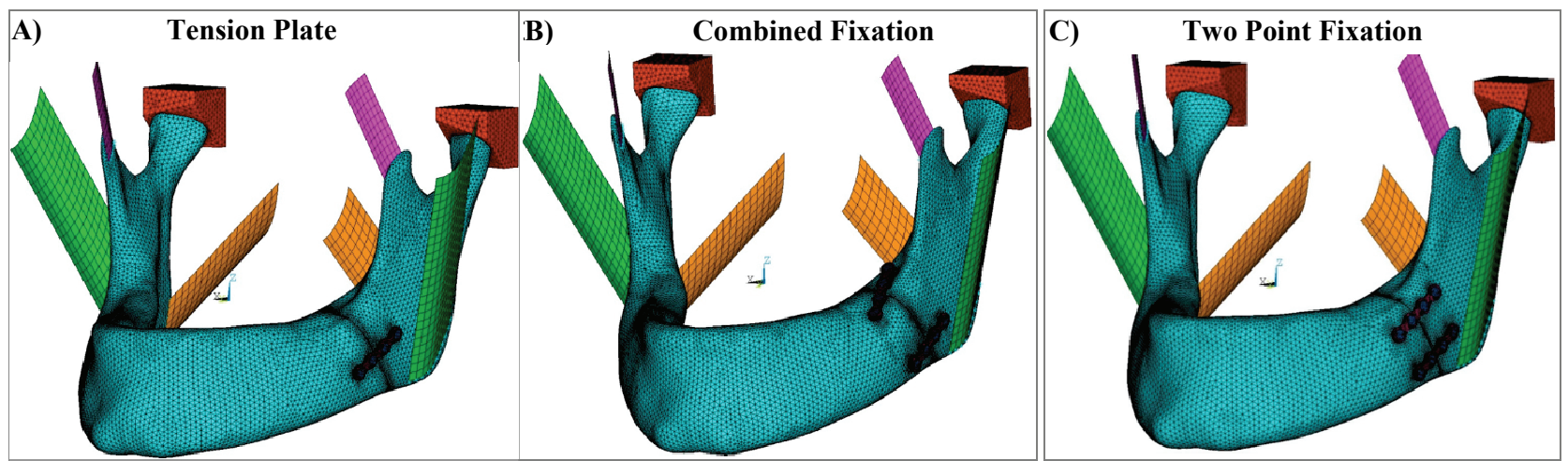

Fig. 3. FEM models of investigated connection types 
A specific nomenclature has been introduced to convenient model identification. The tension plate (Fig. 3A), combined fixation (Fig. 3B) and two-point fixation (Fig. 3C). Mesh model's statistics are included in (Table 2).

Table 2. Mesh models statistics

\begin{tabular}{|l|c|c|c|}
\hline & $\begin{array}{c}\text { Tension } \\
\text { plate }\end{array}$ & $\begin{array}{c}\text { Two point } \\
\text { fixation }\end{array}$ & $\begin{array}{c}\text { Combined } \\
\text { fixation }\end{array}$ \\
\hline Elements & 529086 & 569024 & 460943 \\
\hline Nodes & 733739 & 761274 & 638384 \\
\hline
\end{tabular}

\subsection{Boundary conditions}

To stabilize system and prevent rigid body motion, the TMJ mimic block's translational degrees of freedom have been restrained on two external sides (Fig. 4A). Due to the small stiffness, the flexible artificial block is easily deformed by rotating mandible bone under assumed load without affecting bone stress. This assumption makes it possible to reflect the mandible physiological movement at the same time as simplifying the FEM model, by eliminating two nonlinear contact pairs between the condyle of the mandible, articular disk and temporal bone. The muscle system included in the designed models contains three pairs of main mastication muscles, the masseter, temporalis and medial pterygoid, which stationary ends have been fixed to lock their movement. Direction and location of the particular muscles attachment relative to the mandible were defined basis on their anatomical position [21]. The muscle surfaces were connected

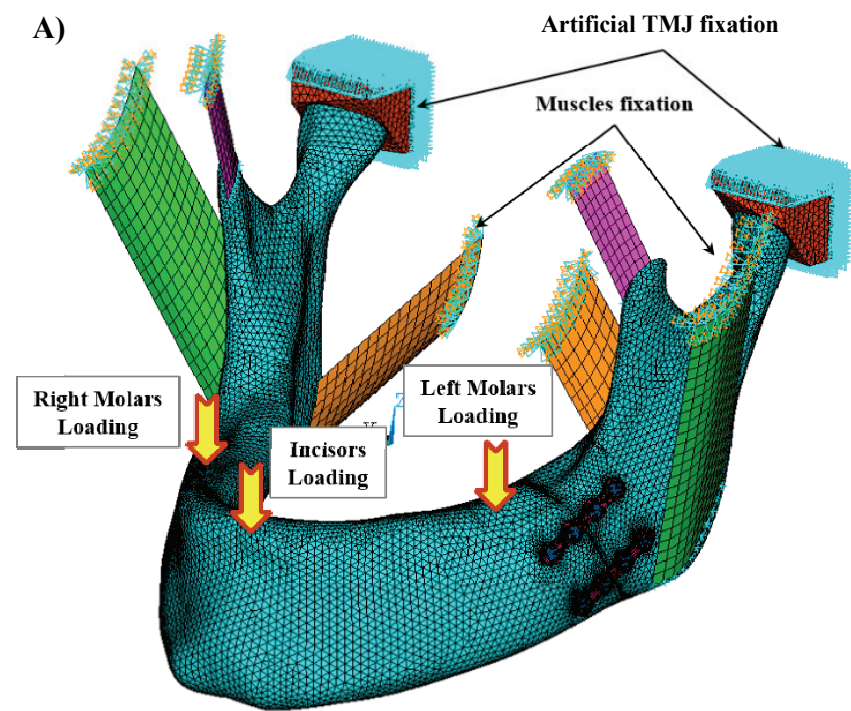

with the mandible bone by the constrain equation contact algorithm. That formulation tied the displacement between contacting surfaces to create the bonded or no-separation contact conditions [1].

The interaction between fractured bones and connecting devices was described by the surface-tosurface frictional contact. The contact analysis introduced nonlinearities to the overall stiffness matrix, making the entire analysis more complex and more sophisticated to converge. The CNOF (contact normal offset) parameter, defined as the offset of entire contact surface, was deployed to simulate the initial gap or compression on the fracture plane instead of modifying geometry.

A positive value of $\mathrm{CNOF}$ shifts the entire contact surface towards the target surface (introducing compression), while a negative offset value creates the gap at the interface (Fig. 4B) [1]. Following the [22], the frictional standard contact with the friction coefficient of 0.3 and the variable CNOF in the range from -1 to $0.5 \mathrm{~mm}$ were applied to the contact pair between broken bone segments on the fracture plane. Application of the positive or negative distance enables simulating the initial compression or a gap between connected bones seen during the fracture treatment, depending on the fracture conditions and shape.

The initial compression is enforced by the special type of compression plates, where immersing screws shift connected bone segments relative to each other (Fig. 5A). The use of initial preload enables us to execute the load-sharing fixation approach, where the masticatory load transfer is shared by the treated bone and the connecting devices (Fig. 5D). This solution is desired to fracture where both ends of broken bone

B)

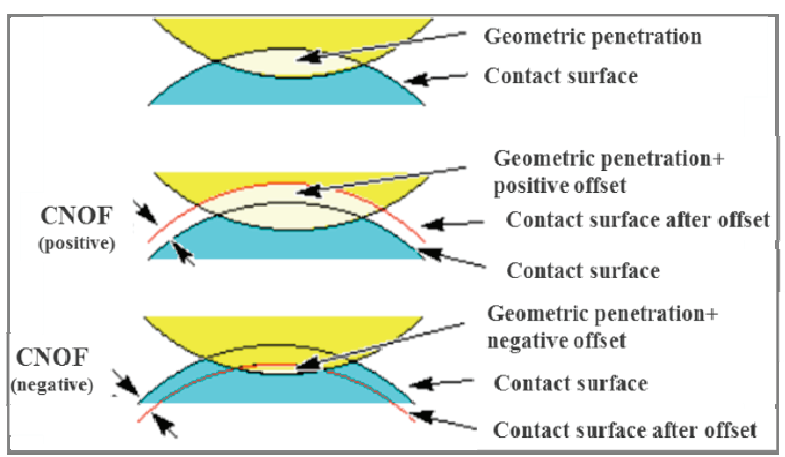

Fig. 4. (A) Loading and model fixation, (B) The physical interpretation of a CNOF parameter [1] 

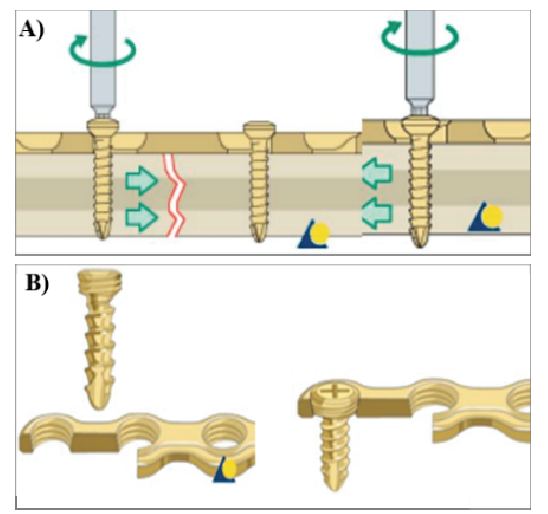
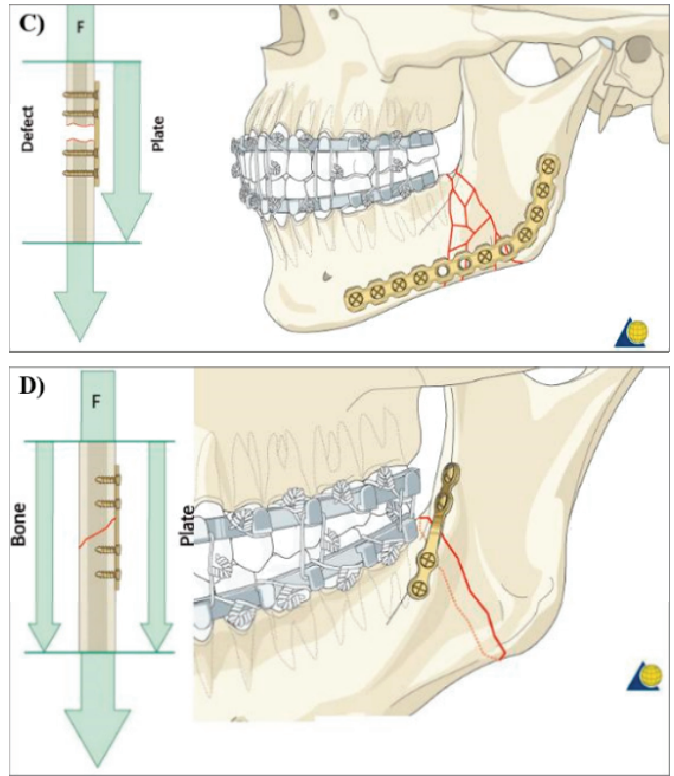

Fig. 5. Compression (A) and locking plate systems (B), load bearing (C) and load sharing (D) [3]

have solid edges with a simple fracture line. This type of connection offers initially compressed rigid fixation of the fracture surfaces but is more difficult to proper establishment.

The gap between bones was executed by sliding fractured mandible halves in opposite direction from the fracture line. That kind of connection is desired to treat the comminuted fracture, where bone is not able to transmit loading. In this case, the loading path went mainly through the connecting devices with very slight contribution of treated bone (Fig. 5C). This phenomenon was described several decades ago, when Worthington and Champy [25] stated the idea of "stress shielding". The aim was that the physiologic stress resulting from the daily loading could stimulate osteogenic cells in two ways, by a piezoelectric or mechanochemical effect. In the case of lack of the physiologic stimulation, a bone loss may be observed. Connecting screws were tied with the surrounding bones by the bonded contact condition (no sliding or separation between surfaces allowed) to reflect behavior of threaded connection described as cylindrical surfaces. The same bonded contact conditions were applied to the interface between screws and miniplates, mimicking the locking plate system, where both, plate holes and screw heads were threaded due to the internal and external fixation. The rigid connection between screws and the plate ensured more stable connection, compared to the non-locking system, also minimizing the risk of screw loosening (Fig. 5B). To prevent the mini-plate from penetrating into the cortical bone and determine proper position relative to the mandible between the plate and bone, another frictional contact condition was set with the friction coefficient of 0.3 [22].

\subsection{Loading variants}

During the daily lifecycle, the mandible bone is mainly loaded by the masticatory bite forces, where the maximum force is estimated to be up to $400 \mathrm{~N}$ for the average healthy man, but significantly reduced by a period of the treatment [2], [14]. Below analyses were conducted under assumptions of static load conditions. For all three models, three test loading locations with the magnitude of $100 \mathrm{~N}$, used also, among others, by [16], were applied. It corresponds to the allowable connection load of the period between second and fourth week after treatment [12]. The force was separately applied to the incisors, right and left molars (Fig. 4A).

\section{Results}

The evaluation of the FEM analysis results was performed with respect to resulting contact status in the fracture plane and equivalent stress according to the Huber-Mises-Hencky hypothesis, within the mini-plates and mandible cortical bone. The foregoing criterion is adequate for the connecting devices and cortical bone, assuming isotropy of their material models. Contact status describes the mutual behavior of contacting surfaces depending on distance, friction and interaction between themselves. We can dis- 
tinguish open state, where no surface interaction exists and closed state, which is about touching surfaces. Closed state is further divided into sticking and sliding with permissible movement. The open state divides also into near and far open contact, depending on the numerical contact properties. Distribution of equivalent stress according to the Huber-Mises-Hencky hypothe- sis within the connecting mini-plates and surrounding cortical bone significantly differs depending on the contact conditions between the connected bones. Overall comparison shown in Figs. 7, 8 was divided into three groups depending on loading location.

The mini-plates and cortical bone equivalent stress peak values compared between all three contact con-

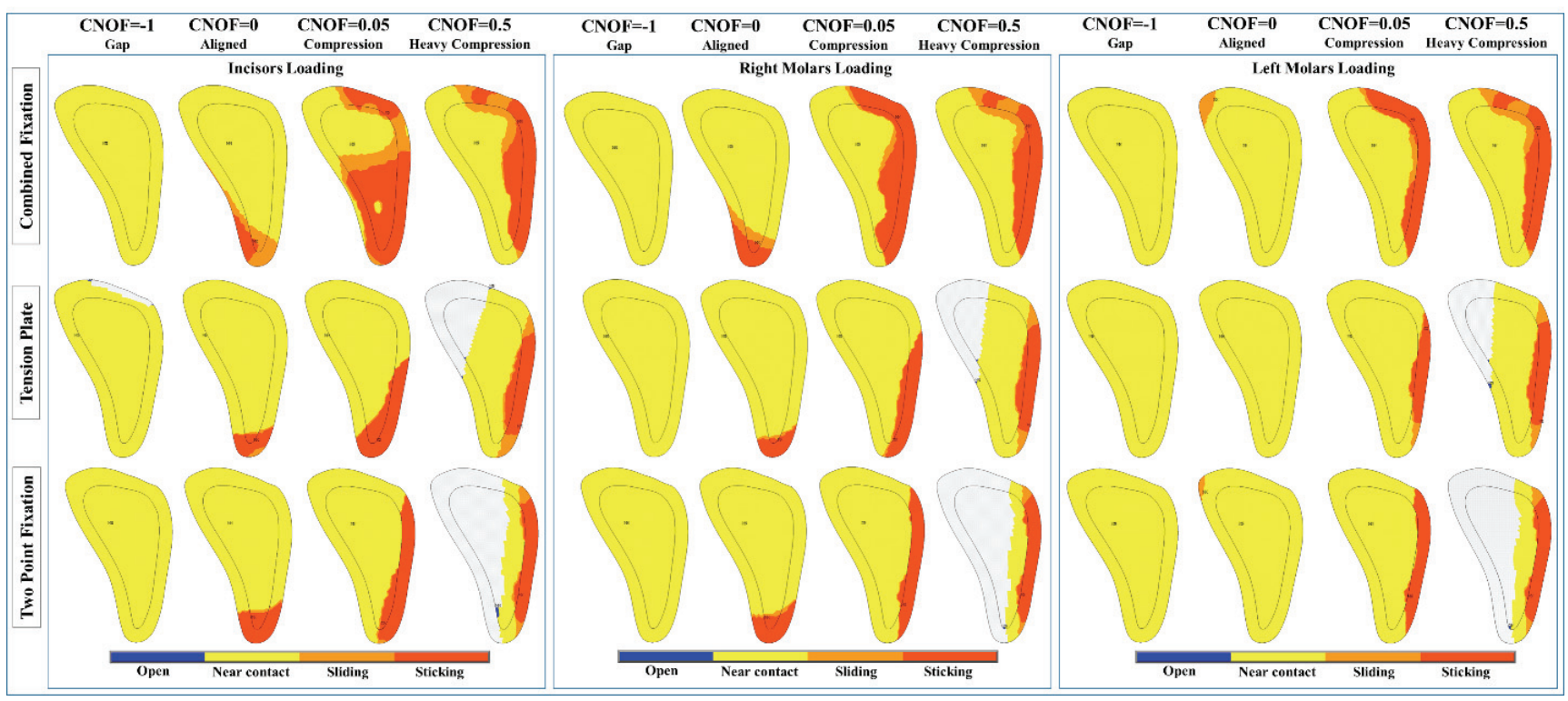

Fig. 6. Mandible fracture contact status for all investigated cases

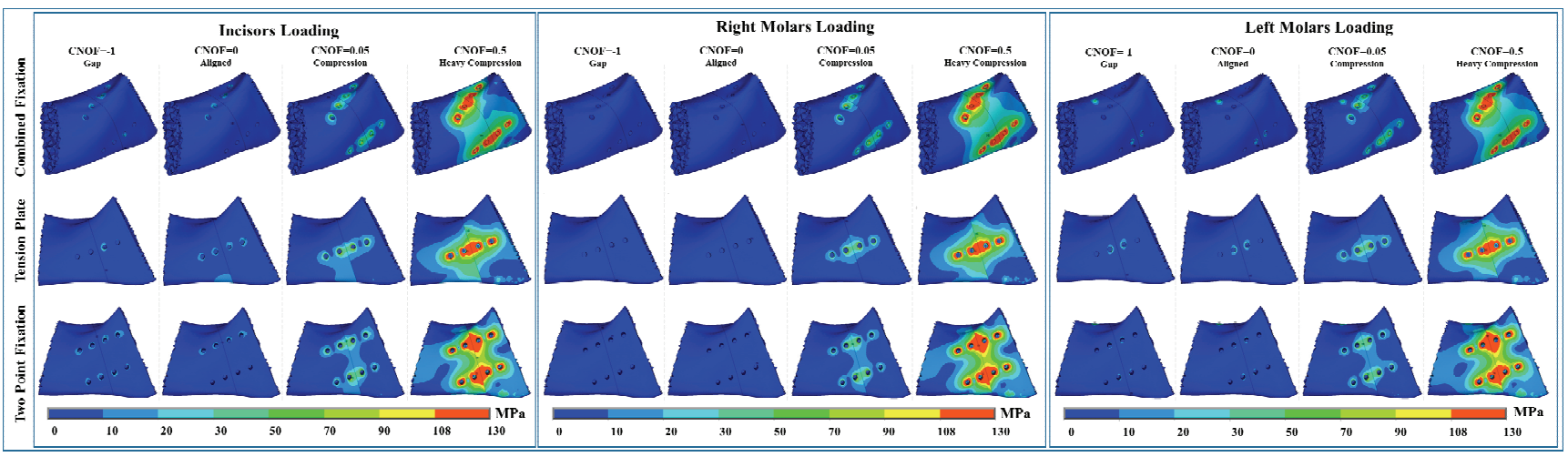

Fig. 7. Equivalent stress distribution within the cortical bone for all investigated cases

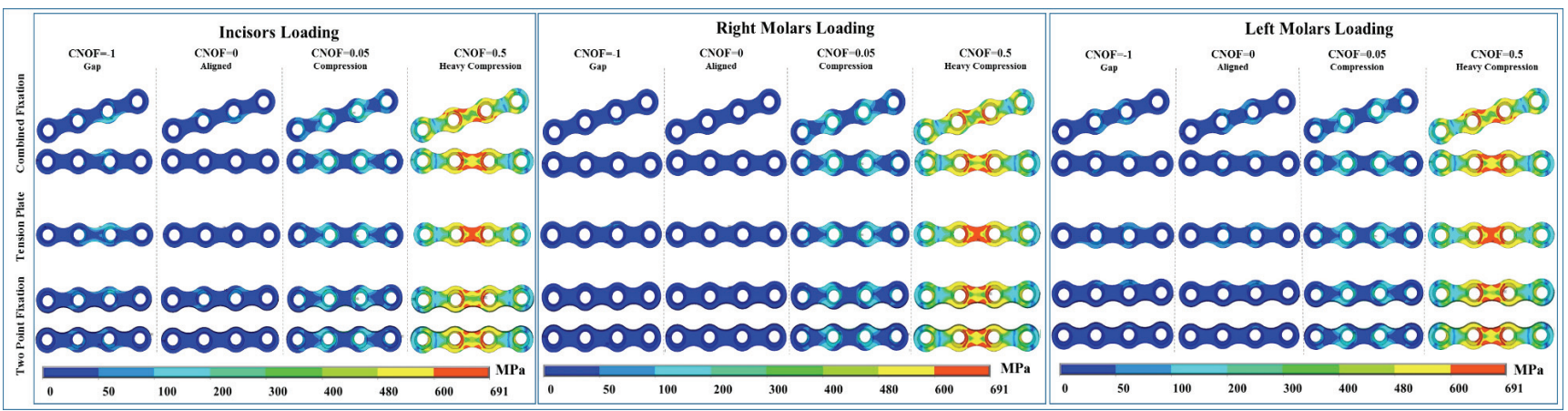

Fig. 8. Equivalent stress distribution within the connecting mini-plates for all investigated cases 
ditions and loading location have been summarized in Figs. 9, 10. To better visualise the differences between connecting patterns and fracture plane contact conditions, the comparisons of cortical bone and mini-plates were presented in the same stress scale range, up to their ultimate tensile strength. Describing stress within the bone, it is really difficult to set clear boundaries between elastic and plastic behavior. Bone can be considered as a fibrous composite consisting primarily of collagen fibers that exhibit the J-shaped stress-strain curve and an inorganic matrix, which can by described by Hookean stress-strain relationship. Maximum bone's total elongation is up to $3 \%$ and, for that reason, it is classified as a brittle rather than a ductile material. Considering both factors, the bone material subjected to loads nearly the yield strength is being destroyed rather than showing plastic deformation. Sometimes fractures occur before reaching the yield range. Following that fact, we can assume that bone's regions indicating the level of stress around the yield limit or higher have been destroyed. Comparing presented contact status and equivalent stress distributions, it is important to keep in mind that resulting numbers are representative for applied load of $100 \mathrm{~N}$.

Due to the bone material properties and eating habits variation throughout the population, from the research point of view, more valuable are the trends or variation of presented variables between proposed connection patterns, load location or fracture plane initial contact state than the exact numeric values.

\section{Discussion}

The conducted analysis has shown the pronounced contact status dependency on the initial contact behavior rather than a connection type or loading location (Fig. 6). Considering the resulting fracture plane contact status, it is important also to look on the final maximum distance between contacting bone surfaces (Table 3). Clinical observation showed that the allowable limit of relative inter-fragmentary motion under the applied load have to be below the $0.15 \mathrm{~mm}$ [2], [14], [19]. The initial distance (gap) applied to the fracture contact pair prevents the bones from touching under assumed loading for all three connection types, regardless of the location of applied force. Additionally, the tension plate pattern presents increased contact separation on upper side due to the notable bone rotation around the middle-located mini-plate, which means that the resulting distance exceeded $0.5 \mathrm{~mm}$. It is not observed at the molars loading patterns due to the lower sagittal bending moment. Despite the bone turning downwards, applied biting force is not able to close the distance to the acceptable limit in the inferior portion of the fracture plane. Resulting distance causes all of the connection patterns work as loadbearing mechanisms.

Liu et al. [19] analyzed the mandible angle fracture treatment with the same connection pattern as the combined fixation, introducing the $1 \mathrm{~mm}$ inter-fragmentary gap. Resulting distances show very similar behavior through the loading locations despite the higher biting forces and different material stiffness and plate geometry.

The aligned contact condition presents interaction of bone fragments at the bottom portion of the fracture plane, but the size of contacting area depends more on the connection type than the load location. It is a direct result of downward bone rotation in the sagittal plane under applied load, while the upper part of fracture plane was opened compared to the initial state.

Similar behavior was observed in the incisors and right molars loading. Loading of the fracture side molars caused total lack of contact except the small region of upper proximal fracture plane side of both double-plate connection patterns. Removal of initial distance between connected bones has changed the load transfer scheme from bearing to sharing type for the cases where physical contact appeared. Maximum resulting contact distance almost fulfilled the requirement for allowable physiologist movement. Arbag et al. [2] conducted analysis on the tension plate and two-point fixation models for aligned contacting surfaces and incisors loading of $100 \mathrm{~N}$. Resulting distance trend aligns with these provided in Table 3 for corresponding models. Higher amplitude they obtained may be caused by slightly different mini-plate design and quality of FEM model, in particular, the

Table 3. Maximum inter-fragmentary bone distance. All distance values are given in [mm]

\begin{tabular}{|l|c|c|c|c|c|c|c|c|c|c|c|c|}
\hline & \multicolumn{4}{|c|}{ Incisors loading } & \multicolumn{4}{c|}{ Right molars loading } & \multicolumn{4}{c|}{ Left molars loading } \\
\hline CNOF [mm] & -1 & 0 & 0.05 & 0.5 & -1 & 0 & 0.05 & 0.5 & -1 & 0 & 0.05 & 0.5 \\
\hline Combined Fixation & 0.38 & 0.08 & 0.03 & 0.28 & 0.36 & 0.10 & 0.10 & 0.32 & 0.48 & 0.12 & 0.22 & 0.40 \\
\hline Tension Plate & 0.52 & 0.12 & 0.09 & 0.64 & 0.48 & 0.15 & 0.26 & 0.67 & 0.49 & 0.17 & 0.32 & 0.69 \\
\hline Two Point Fixation & 0.46 & 0.06 & 0.12 & 0.68 & 0.42 & 0.09 & 0.11 & 0.69 & 0.44 & 0.14 & 0.23 & 0.72 \\
\hline
\end{tabular}


contacts settings. Joshi and Kurakar [14] analyzed the same monoplanar connection patterns as well, with the same material properties as [2] and load amplitude of $62.8 \mathrm{~N}$, but achieved much lower resulting distance. The additional benefit may come from the setting the fracture plane at 45 degree angle relative to the mini-plates, increasing the possible contacting surface. None of them provided data about the friction conditions on the fracture surface if it exists at all, and which significantly affects the fracture mobility.

The initial compression applied to the contact pairs significantly increased the contact area, expanding it mainly onto the distal part of the fracture plane for all three connection patterns. Significant improvement of physical contact is exhibited by the combined fixation model. Bigger contact area is desirable from the treatment point perspective due to the better micromechanical stimulation of bone. However, the initial compression presented better fracture plane performance. The heavy compression reduced the overall effect of light compression by decreasing the contact area on the proximal side due to the rotation in the transverse plane. For both mono-planar connection patterns it resulted in bigger bone separation than the gap condition. Over-tightened initial state may generate locally high contact pressure, which finally may lead to bone degradation and possible perfusion issues. The available literature does not provide computational examples with initial compression for mandible angle fracture, so provided results could be considered as a reference for further investigation.

Equivalent stress distribution observed within the cortical bone, for the gap contact condition, covers only small area around the screw holes, regardless of the connection pattern and load location due to the lack of interaction in the fracture plane. The maxi- mum magnitude of equivalent stress is observed on the incisor load pattern due to the highest sagittal bending moment resulting from the lever arm length, while the combined fixation and tension plate shows similarity in the stress peak values (Fig. 9). Combined fixation presents balanced contribution of all screws in load transfer, what finally results in the lowest peak stress value. Equivalent stress distribution within the mini-plates show similar behavior as in the cortical bone (Fig. 10). Similarity between tension plate and biplanar combined fixation models may be caused by very negligible contribution to the load transmission of the inferior border plate. Following those results, the lower plate may be removed to reduce the overall invasiveness. A very small amplitude of stress is observed within the cortical bone and mini-plates under the right molars loading. That indicates that there is negligible participation of fractures side in the load transfer during loading the opposite (healthy) side of the mandible bone. Left molars load, acting very close to the mini-plate location, produced mainly the shear instead of bending moments. The weakest tension plate model showed the highest stress around the screws next to the fracture plane. The other two models present very similar behavior, but with lower magnitude. Regarding the mini-plates maximum peak stress value is observed on the oblique line plate of combined fixation model. Proximity of the load application may amplify stress within mini-plate, although it is not visible on the surrounding cortical bone. All of the connection patterns show insignificant contribution of outer holes to load transfer.

Liu et al. [19] provided peak stress magnitudes for the cortical bone and mini-plate's material for analyzed "type B" connection pattern. The equivalent stress distribution within cortical bone shows inverse be-

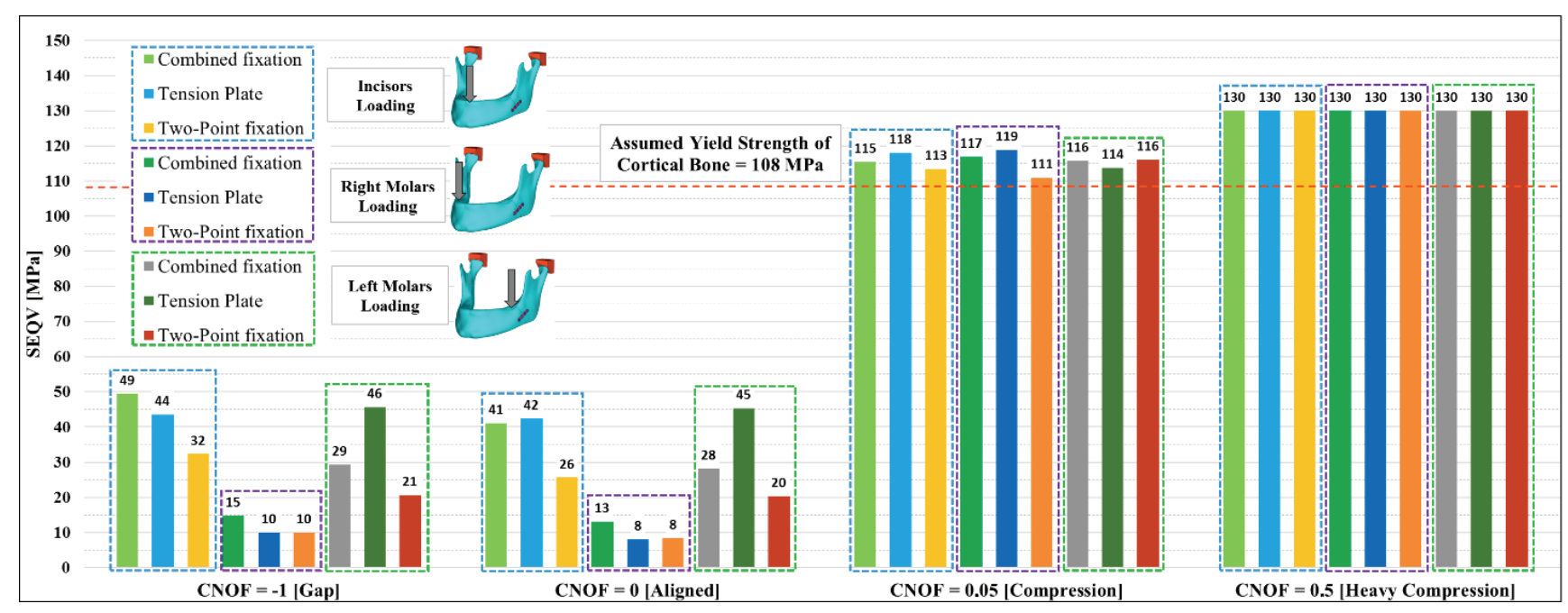

Fig. 9. Maximum equivalent stresses observed within the cortical bone at all analyzed cases 


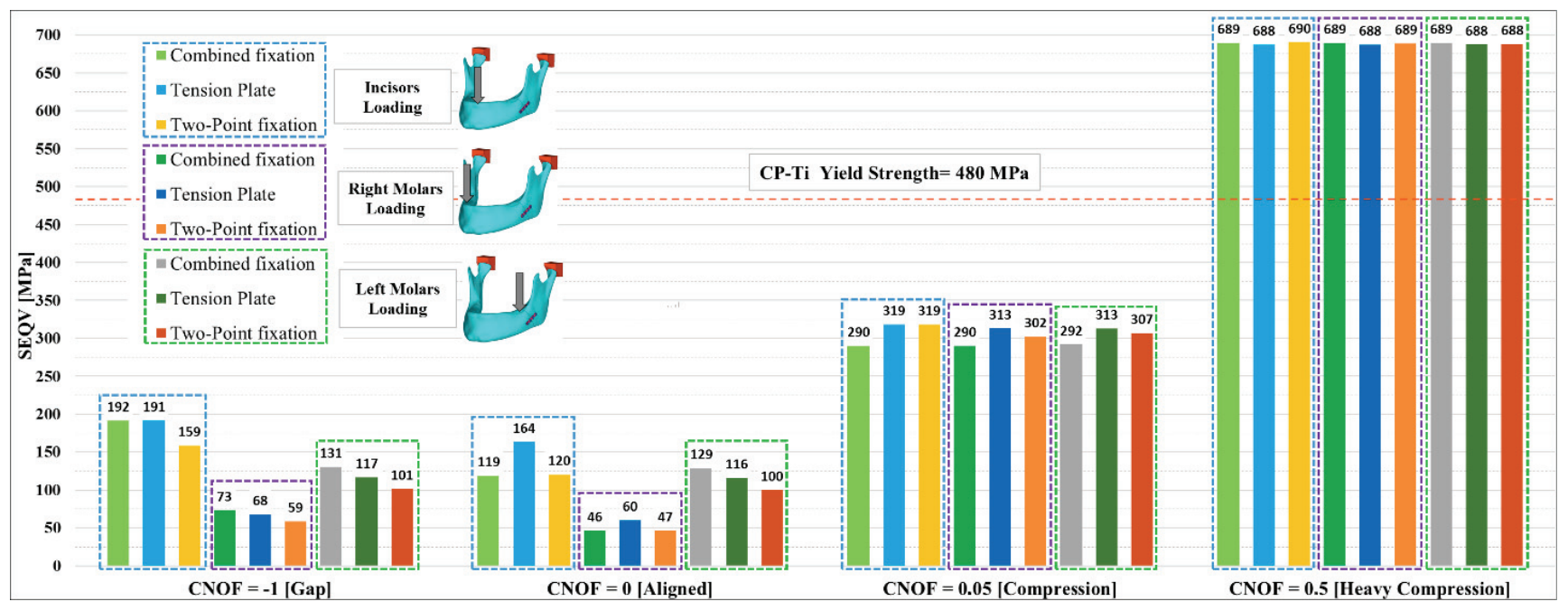

Fig. 10. Maximum equivalent stresses observed within connecting mini-plates for all analyzed cases

havior to results provided herein. Maximum stress is observed on the coronoid process, during loading the healthy mandible side. Regarding the incisors and healthy side loading, the critical location is observed around the screw hole. Several factors may impact these results, but most doubtful is the quality of FEM model, especially in the critical fastener's regions. As it is acceptable from the displacement point of view, the stress requires much more attention. Despite the materials, loading and quality differences the miniplate stress behavior is similar, showing the maximum while loading the fracture side.

Adjusting contact sides has decreased the peak stress magnitude achieved during the incisors load within the cortical bone and mini-plates. This stress reduction is caused by the transition from bearing to sharing load transfer path. Interaction area between mini-plates and treated bone is observed on the inferior portion of fracture plane (Fig. 6).

The tension plate model presents the $15 \%$ reduction of the peak stress and is the least sensitive pattern to the load sharing due to the lower stiffness of entire system. It results in creation of the increased stress area in the inferior mandible body region due to the notable bone rotation under applied force (Fig. 7). Regarding the combined and two-point fixation models, the equivalent stress within the cortical bone was reduced by $17 \%$ and $19 \%$, respectively. Observed equivalent stress reduction, within mini-plates ranges between $15 \%$ for tension plate to $38 \%$ for combined fixation (Figs. 9, 10). All connecting devices exhibit the reduction of higher stress regions, mainly at the inferior plates of double braced connections, which have been partially unloaded by the contacting cortical bone (Fig. 8). Right molars load case showed overall stress decrease within the cortical bone by around $20 \%$.
Presented decrease is connected with progressing bones contact around the inferior border line as mentioned previously. Most of loads are still transmitted by the healthy side of mandible. Regarding the miniplates, the equivalent stress reduction is between 12 and $27 \%$, while the biggest improvement is visible on the combined fixation model. Tension plate pattern shown the highest peak stress, same as under the incisors loading scheme. There are negligible differences in peak stress value between aligned and gap initial contact conditions under the left molars loading case. Both, cortical bone and mini-plates consistently shown reduction by around $1 \mathrm{MPa}$ for all three connection patterns (Figs. 9, 10). Overall stress distributions also remain the same (Figs. 7, 8). Arbag et al. [2] obtained the equivalent stress within connecting miniplates for incisor loaded mandible. The peak values are more than twice bigger despite smaller load amplitude. Lower material stiffness may also affect the reported readings. Equivalent stress relation between two and single mini-plates patterns show about $25 \%$ reduction in the case of double connection, what is in line with described analysis. That indicates that, in macro scale, both systems seem to be similar regardless of modeling differences.

Compression introduced to the fracture plane increased observed stress magnitude within both, the mini-plates and cortical bone. Maximum cortical bone equivalent stress has reached the level of possible bone degradation, which may be observed in limited area around the screw holes. Local stress peaks may be vanished by the stress relaxation at the later stage of the treatment. In this case, there is no notable difference in equivalent stress peak values between load location and connection pattern within mini-plates, although, combined fixation presented slightly lower 
peak stress within the mini-plates compared to other models. This kind of initial contact condition seems to be promising in terms of the contact area and stresses within presented components.

Heavy compression amplified stresses to the yield material limit within the cortical bone. Area at risk of destruction covered almost entire area between all four screws, which finally may result in screw loosening. Regardless of connection type or applied force location, all analyzed mini-plates exceeded the yield strength of CP-Ti. Plastic deformation appeared in the neck between the inner holes, dramatically reduced the material strength capacity (Figs. 7, 8). Observed system's load response eliminated the heavy compression from the promising and safe treatment approach in terms of the stress within the analyzed components. It is possible to determine the limiting compression rate in a computer-based study, but it is difficult to assess it for clinical conditions, often with complicated fracture line with jagged ends, which reduce the fracture mobility.

Compared to similar analysis available in the literature, provided results show similar behavior regarding the maximum displacements. There was no information about displacement distribution within the fracture plane after loading (represented by the contact status), which clearly shows the exact behavior of connecting system and the scale of possible nonacceptable separation. Equivalent stress presented by legacy papers deviates from presented results for several reasons, but it seems that the too rough modeling might be the main cause. Assumed simplification regarding the TMJ modeling was not improperly affecting conducted analysis. It was proven that it could be a good approach to back-to-back analysis, where the joint is not an object of interest.

Also, for the comparison purposes, the bone materials isotropy seems to be acceptable, as long as the detailed stress distribution is not required. Precise FEM model prepared for presented analysis provides acceptable fidelity in the fasteners regions basis on the stress gradients and behavior of the contact itself. The concept of mini-plates was to provide the functional stability, where some micro-motions are desirable to stimulate the bone treatment process. Neither too wide clearance nor heavy compression would provide that basic functionality. Accurate number and locations of mini-plates may significantly reduce the bending moments during the daily mandibular function. The miniplate failure has to be prevented during the treatment period as well. As presented results show, overloading compressive connection pattern leads to exceeding of the material strength limit. Daily masticatory forces generate additional spectrum of alternating load, which may be important in terms of the fatigue endurance. The higher static stress generated by initial conditions, the lower fatigue stress margin remains. Considering variable masticatory load amplitudes, the assessment of accumulated fatigue cycles is very sophisticated, almost impossible. That's the reason of why the static stress within both, cortical bone and mini-plates have to be as low as possible during the initial mini-plate installation to keep the safety margin for overall possible load spectrum.

\section{Conclusions}

Summarizing, the above study evaluated the impact of mini-plate fixation type, fracture plane contact conditions and loading location on the resulting fracture plane contact condition and equivalent stress within cortical bone and connecting devices. Three detailed FEM models were generated to resemble the mandible - plates behavior under assumed biting force.

The findings of present study suggest that the biplanar combined fixation pattern under the slight initial compression is the most promising connection approach. Observed the fracture plane contact behavior, maximum inter-fragmentary distance and equivalent stress distributions are acceptable from the biomechanical point of view. The plate application on the oblique line relatively increases the connection invasiveness but is outweighed by better connection stability. The numerical simulation, as long as it concerns the living structures, has certain limitations. Mandible angle region presents a complex problem from the mechanical point of view, but, for the comparison reasons, we can assume that the general understanding of behavior of the provided connections have been exposed. Incorporated contact offset enabled us to easily mimic different load transmission patterns. Calculation conducted on the same discrete model saved time of additional model preparation and increased overall analysis accuracy. However, extra effort must be invested to develop the mandible's orthotropic material properties in the case of need to compute the exact strength and possible fatigue life. The biomechanics analysis result is one of several factors which have to be considered during the fracture treatment. It is impossible to simulate all clinical cases, but it is important to use the simulation results as a basis for the treatment strategy and consider involved complications. 


\section{Acknowledgements}

This research was supported by The Research Fund of the Dean of the Faculty of Power and Aeronautical Engineering of Warsaw University of Technology.

\section{References}

[1] ANSYS HELP, ANSYS Inc., Release 17.1.

[2] Arbag H., Korkmaz H.H., OzTurk K., UYAR Y., Comparative Evaluation of Different Miniplates for Internal Fixation of Mandible Fractures Using Finite Element Analysis, Journal of Oral and Maxillofacial Surgery, 2008, 66 (6), 1225-1232.

[3] Arbeitsgemeinschaft für Osteosynthesefragen, Surgery reference.

[4] Atlas of stress-strain curves (2nd ed.), ASM International, Materials Park, OH, 2002.

[5] BAŃCZEROWSKI J., WĄDOŁOWSKI P., KRZESIŃSKI G., GUTOWSKI P., Modelling and strength analysis of a mandible miniplate, Surface Engineering, IMP Warsaw, 2016, 21 (1), $30-40$.

[6] Boffano P., Roccia F., Zavattero E., Dediol E., Uglesic V., Kovacic Z., Vesnaver A., Konstantinovic V.S., Petrovic M., Stephens J., Kanzaria A., Bhatti N., Holmes S., Pechalova P.F., BaKardjiev A.G., MalanchuK V.A., KopchaK A.V., Galteland P., Muoen E., Skjelbred P., Grimaud F., Fauvel F., Longis J., Corre P., Loes S., LeKVEn N., LAVERICK S., Gordon P., TAMme T., AKermann S., Karagozoglu K.H., Kommers S.C., MeiJer B., FOROUZANFAR T., European Maxillofacial Trauma (EURMAT) in children: A multicenter and prospective study, Oral Surgery Oral Medicine Oral Pathology Oral Radiology, 2015, 119 (5), 499-504.

[7] Boyer R., Welsch G., Collings E.W., Materials Properties Handbook: Titanium Alloys, ASM International, Materials Park, $\mathrm{OH}, 1994$.

[8] Bujtar P., Simonovics J., Varadi K., SAndor G.K.B., AVERY C.M.E., The biomechanical aspects of reconstruction for segmental defects of the mandible: A finite element study to assess the optimization of plate and screw factors, Journal of Cranio-Maxillofacial Surgery, 2014, 42 (6), 855-862.

[9] Champy M., Lodde J., Schmitt R., Jaeger J., Muster D., Mandibular Osteosynthesis by Miniature Screwed Plates Via a Buccal Approach, Journal of Maxillofacial Surgery, 1978, $6(1), 14-21$.

[10] ChOI A., Ben-Nissan B., Conway R., Three-dimensional modelling and finite element analysis of the human mandible during clenching, Australian Dental Journal, 2005, 50 (1), 42-48.

[11] Ding X., LiaO S., Zhu X., Wang H., Zou B., Effect of orthotropic material on finite element modeling of completely dentate mandible, Materials and Design, 2015, 84, 144-153.
[12] Harada K., Watanabe M., Ohkura K., Enomoto S., Measure of bite force and. occlusal contact area before and after bilateral sagittal split ramus osteotomy of the mandible using a new pressure-sensitive device: A preliminary report, Journal of Oral and Maxillofacial Surgery, 2000, 58 (4), 370-373.

[13] ICHIM I., KIESER J.A., SwaIn M.V., Functional significance of strain distribution in the human mandible under masticatory load: Numerical predictions, Archives of Oral Biology, 2007, 52 (5), 465-473.

[14] Joshi U., KURAKar M., Comparison of Stability of Fracture Segments in Mandible Fracture Treated with Different Designs of Mini-Plates Using FEM Analysis, Journal of Maxillofacial and Oral Surgery, 2014, 13 (3), 310-319.

[15] Kromka M., Milewski G., Experimental and numerical approach to chosen types of mandibular fractures cured by means of miniplate osteosynthesis, Acta Bioeng. Biomech., 2007, 9 (2), 49-54.

[16] Kromka-Szydek M., Jędrusik-PawŁowska M., Milewski G., LeKSTON Z., CIEŚLIK T., DRUGACZ J., Numerical analysis of displacements of mandible bone parts using various elements for fixation of subcondylar fractures, Acta Bioeng. Biomech., 2010, 12 (1), 11-18.

[17] Langenbach G., Hannam A., The role of passive muscle tensions in a three-dimensional dynamic model of the human jaw, Archives of Oral Biology, 1999, 44 (7), 557-573.

[18] LEE K., Global trends in maxillofacial fractures, Craniomaxillofacial Trauma Reconstruction, 2012, 5, 213-222.

[19] LiU Y., FAn Y., Jiang X., Baur D.A., A customized fixation plate with novel structure designed by topological optimization for mandibular angle fracture based on finite element analysis, BioMedical Engineering OnLine, 2017, $16,131$.

[20] O’Mahony A., Williams J., Katz J., Spencer P., Anisotropic elastic properties of cancellous bone from a human edentulous mandible, Clinical Oral Implants Research, 2000, 11 (5), 415-421.

[21] PUtz R., Pabst R., Atlas Sobotta Atlas der Anatomie des Menschen, Elsevier GmbH, Munich 2006.

[22] Ramos A., Duarte R.J., Mesnard M., Prediction at long-term condyle screw fixation of temporomandibular joint implant: A numerical study, Journal of Cranio-Maxillofacial Surgery, 2015, 43 (4), 469-474.

[23] Schwartz-Dabney C., Dechow P., Variations in cortical material properties throughout the human dentate mandible, American Journal of Physical Anthropology, 2003, 120 (3), 252-277.

[24] TORREIRA M., Fernandez J., A three-dimensional computer model of the human mandible in two simulated standard trauma situations, Journal of Cranio-Maxillofacial Surgery, 2004, 32 (5), 303-307.

[25] Worthington P., Champy M., Monocortical Miniplate Osteosynthesis, Otolaryngology Clinics of North America, 1987, 20 (3), 607-620. 